\title{
P01-13-4 Poster session
}

\section{Selection of spinal needles for cerebrospinal fluid collection in clinical trials}

Tatsuya Yoshihara ${ }^{1}$, Koki Furusho ${ }^{1}$, Yukikuni Sakata ${ }^{1}$, Hiroshi Tsukikawa ${ }^{1}$, Ryuzo Hanada ${ }^{2}$, Rie Yazawa ${ }^{2}$, Takeshi Chiyoda ${ }^{2}$, Ippei Ikushima ${ }^{2}$, Shunji Matsuki ${ }^{1}$, Shin Irie ${ }^{1}$

${ }^{I}$ SOUSEIKAI Fukuoka Mirai Hospital, Clinical Research Center, Japan, ${ }^{2}$ SOUSEIKAI Sumida Hospital, Japan

Background:

The need of cerebrospinal fluid (CSF) collection is increasing in psychoactive drug development. Postdural puncture headache (PDPH) is the most common complication after the procedure. Thinner needles and/or non-cutting needles (called pencil-point or Sprotte needle) are often used to prevent PDPH. But obtaining necessary amount of CSF is also crucial in clinical trials. Generally, thinner needles need more time to collect CSF than thicker needles, therefore selection of needles is a key factor for successful trials. In this study, we retrospectively analyzed the outlet speed of CSF by needle type.

Methods:

The data of 44 volunteers (age, $70 \pm 4$; male, 59\%) who underwent CSF collection from September 2016 to December 2017 at SOUSEIKAI Fukuoka Mirai Hospital were analyzed. We changed the needle types to prevent headaches: first, we used 21G non-cutting (Quincke) needles, then changed to 22G Quincke needles, and we now use 22G pencil-point needles for elderly volunteers.

Results:

21 G Quincke needles were used for 26 volunteers and 22G needles for 18 volunteers (Quincke needles for 6 and pencilpoint needles for 12). Although mean BMI was higher in the $21 \mathrm{G}$ group than the $22 \mathrm{G}$ group (23.7 \pm 2.0 vs $21.9 \pm 3.0$, $\mathrm{p}=0.02)$, time from local anesthesia to CSF outflow start was not different between the $21 \mathrm{G}$ and $22 \mathrm{G}$ groups $(3.7 \pm 3.1$ vs $3.7 \pm 1.7 \mathrm{~min}$, respectively). The CSF outflow speed was faster in the $21 \mathrm{G}$ group than the $22 \mathrm{G}$ group $(2.0 \pm 0.5$ vs $1.3 \pm$ $0.3 \mathrm{~mL} / \mathrm{min}$, respectively, $\mathrm{p}<0.0001$ ). Types of needle in the $22 \mathrm{G}$ group (Quincke or pencil-point needle) did not affect the outflow speed $(1.3 \pm 0.4$ vs $1.3 \pm 0.3 \mathrm{~mL} / \mathrm{min}$, respectively). Two volunteers $(6 \%)$ had headaches and $2(6 \%)$ had heaviness of head in the Quincke needle group, but no volunteer $(0 \%)$ had headaches or heaviness of head in the pencilpoint needle group.

Conclusions:

CSF outflow speed was slower with thinner needle. To prevent PDPH and obtain required amount of CSF, needle type should be carefully selected based on the amount of necessary CSF, acceptable time in lateral position for volunteers and the incidence rate of complication. 